MULTIFREQUENCY OBSERVATIONS OF BLAZARS

\author{
E I ROBSON, W K GEAR \& L M J BROWN \\ School of Physics \& Astronomy \\ Lancashire Polytechnic \\ Preston PRI 2TQ UK
}

\title{
Abstract
}

This paper reports the results of a programme of multifrequency observations of a sample of Blazars concentrating particularly on the millimetre through infrared region. Our observations have demonstrated the need for a new model to explain the spectral behaviour of a major flare in $3 \mathrm{C} 273$. This also provides a good description of the variability exhibited by Blazars. We have also shown that the radio through mid-IR synchrotron spectrum of $3 \mathrm{C} 273$ is a separate component from the optical/IR.

The Programme of Observations

Although observations in the submillimetre wavelength regime are difficult they are very important (1) because it is here that the transition from the flat radio spectrum to the steep IR/optical spectrum occurs. A determination of the turnover frequency, $v_{m}$, allows physical conditions of a synchrotron emitting region to be calculated. Furthermore, observations of how the optically thin submm/IR and the flat millimetre-wave optically thick component develop as a function of time are necessary to test the viability of emission models (see below). Our first results (2) showed that almost all the sources remained flat up to $1 \mathrm{~mm}$. We detected the superluminal quasar 3C273 at $350 \mu \mathrm{m}$ and using our data combined with observations at other wavelengths, we made the first determination of the overall radio through optical spectrum of a quasar (3). The smooth spectrum was very suggestive of a single synchrotron component covering the millimetre through optical regions but our recent observations have shown that the spectrum is more complex. For the multifrequency programme the 1-3300 pm observations were made using UKIRT and the NRAO $12 \mathrm{~m}$, radio from the VLA, optical from the Florida group and CCD service observations at La Palma, UV from IUE, and $\mathrm{X}$-ray from EXOSAT.

Our sample was selected using specific criteria, namely: the source was a BLLac or OVV quasar; the $1 \mathrm{~mm}$ flux density extrapolated from the radio was $\mathrm{S}_{v}>1 \mathrm{Jy}$; the source must be observable from UKIRT $\left(-38^{\circ}<\delta<59^{\circ}\right)$. This gave a sample of 22 sources, of which 14 were selected for further 


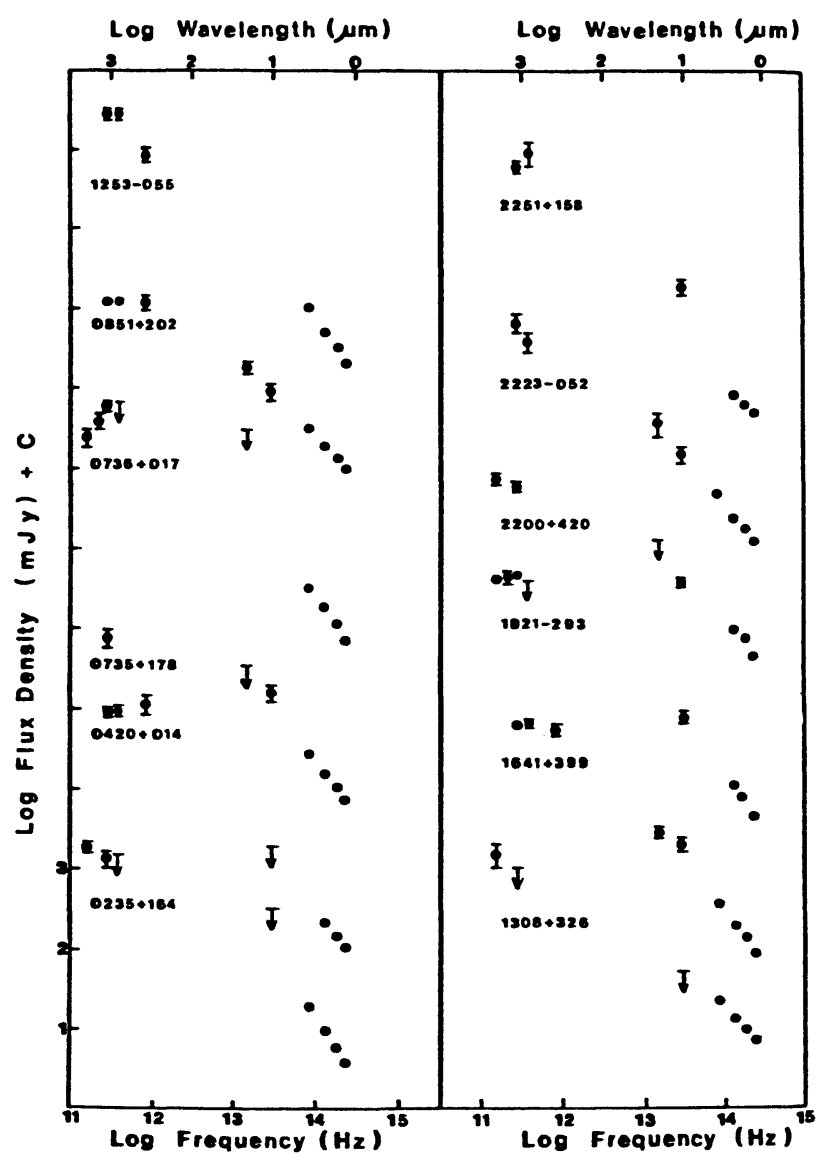

Fig 1. $1 \mathrm{\mu m}$ to $3.3 \mathrm{~mm}$ 'Snapshot' spectra of a sample of 12 Blazars from Gear et al (4).

study. These were: the BLLacs $0235+164,0420-014,0735+178,0851+202$ (OJ287), 1308+326, 1413+135, 1921-293 (OV236) and 2200+420 (BLIAC); the OVV's 0736+017, 1253-055 (3C279), 1641+399 (3C345), 2223-023 (3C446) and $2251+158$ (3C454.3). Two superluminal quasars were also added to the programme (3C120 and 3C273) plus NGC1275.

The results of 'snapshot' measurements of the IR through mm continuum are presented in Gear et al (4) and are summarised by:(a) the IR and submm spectra are characterised by power laws, (b) the infrared spectral indexes of the 12 most studies Blazars ranged from -0.9 to -1.6 with a mean of $-1.25 \pm 0.2$, (c) there was no evidence of any contribution from thermal emission from heated dust, (d) the submm spectrum is generally flat to the shortest wavelengths $(800$ or $350 \mu \mathrm{m})$ but two sources (1253-055 and 2223-052) clearly reveal the presence of a submm turnover $\left(\nu_{m}\right)$, the spectrum steepening at shorter wavelengths, (g) 0736+017 and 1921-293 (5) showed evidence typical of synchrotron self-absorption at about $1 \mathrm{~mm}$. Examples of the 'snapshot' spectra are shown in Fig 1. 
We choose to interperet the overall $\mathrm{mm}$ through IR continum spectrum as being due to synchrotron emission from an unresolved relativistic jet viewed close to the line of sight, which fits in well with other observational inferences. The spectra were well fitted by such a model and physically reasonable values for the core sizes $\left(10^{-3}\right.$ to $\left.10^{-4} \mathrm{pc}\right)$, magnetic fields $(0.1 \mathrm{G})$ and electron densities $\left(10^{8}\right.$ to $\left.10^{10} \mathrm{~cm}^{-3}\right)$ were derived. Luminosities ranging from $3 \times 10^{11}$ to $4 \times 10^{14} \mathrm{~L}_{\odot}$ were calculated assuming isotropic emission $\left(\mathrm{q}_{0}=0, \mathrm{H}_{0}=100\right)$ and in the absence of $a$ strong X-ray component these are close to the bolometric luminosities of the sources (although beaming would reduce these values. To determine more about the physical processes of the emission mechanisms it is necessary to progress beyond 'snapshot spectra' to evolutionary studies. It is good to see at least a few groups pursuing this challenging field of studies (eg 6 and refs therein).

The first results of the variability studies of 12 sources from the sample are published in (7). The results from our studies using the entire wavelength database are given in (8) and an example is shown in Fig 2. We have found that all the sources are variable both in flux and spectral shape with the degree of variability increasing with increasing frequency. We also observe that the IR fluxes increase in unison at all frequencies on a timescale of months or less, thereby implying that this emission arises in a single region. The timescale for the submm/mm variations appears to be longer than for the IR. It is also apparent that a general picture is emerging whereby the infrared spectrum

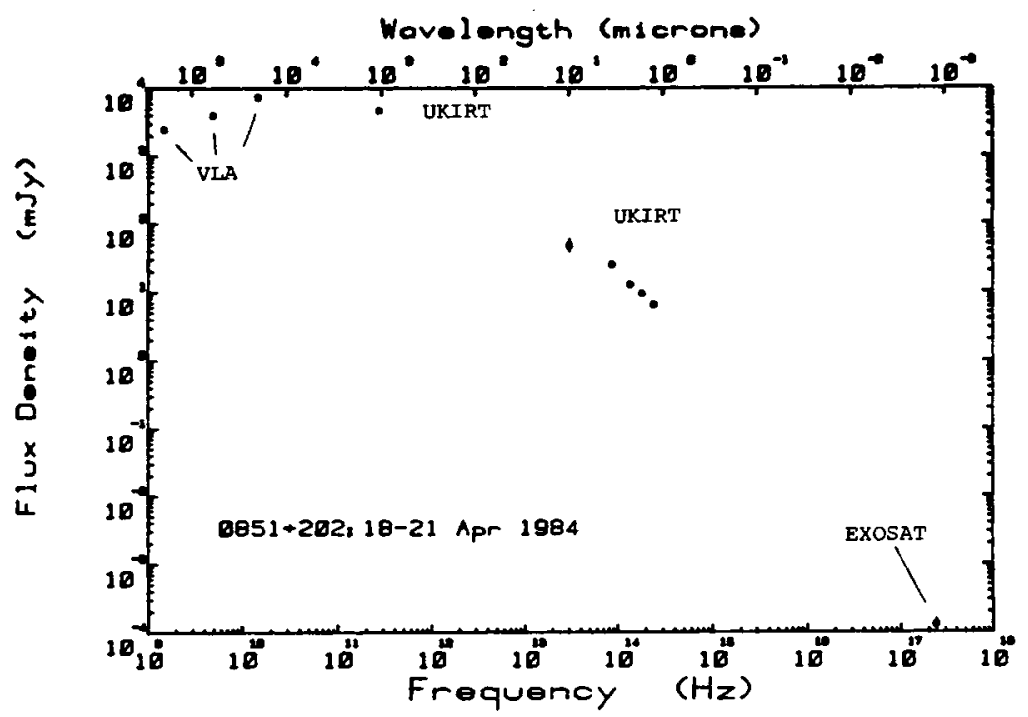

Fig 2. Radio through X-ray 'simultaneous' observations of the BL Lac object $0851+202$ (0J287). The rapid variability of these objects is demonstrated by the fact that the IR and $\mathrm{X}$-ray fluxes had faded by factors of 5 and 10 over 2 months. 
flattens when the infrared fluxes increase and then steepens as the flux decays (9). This suggests that an increase in emission is due to an injection or reacceleration of electrons having a flatter energy distribution. These electrons suffer radiative losses on a timescale of weeks or less giving rise to the resulting spectral decay and steepening.

We have observed comprehensively three sources $(0851+202,1253-055$ and $1641+399)$ in all wavebands on more than one occasion. These all showed evolution from having a flat submm spectrum to a steeper spectrum, (Fig 3 ). The evolution of the submm spectra of these three sources suggest that they have experienced a transition from optically thick to thin emission. In each case there is a simultaneous decrease in the IR fluxes as would be expected from an expanding region. However there is not the expected decrease in flux near the turnover which such a model would suggest. In fact the turnover flux appears to have increased while moving to longer wavelengths, thereby violating the predictions of expanding clouds. This behaviour is very similar to that seen in the large flare of 3C273 which was successfully modelled by Marscher and Gear (10). In this model, the IR through submm emission arises in a small region behind a shock wave travelling in an unconfined

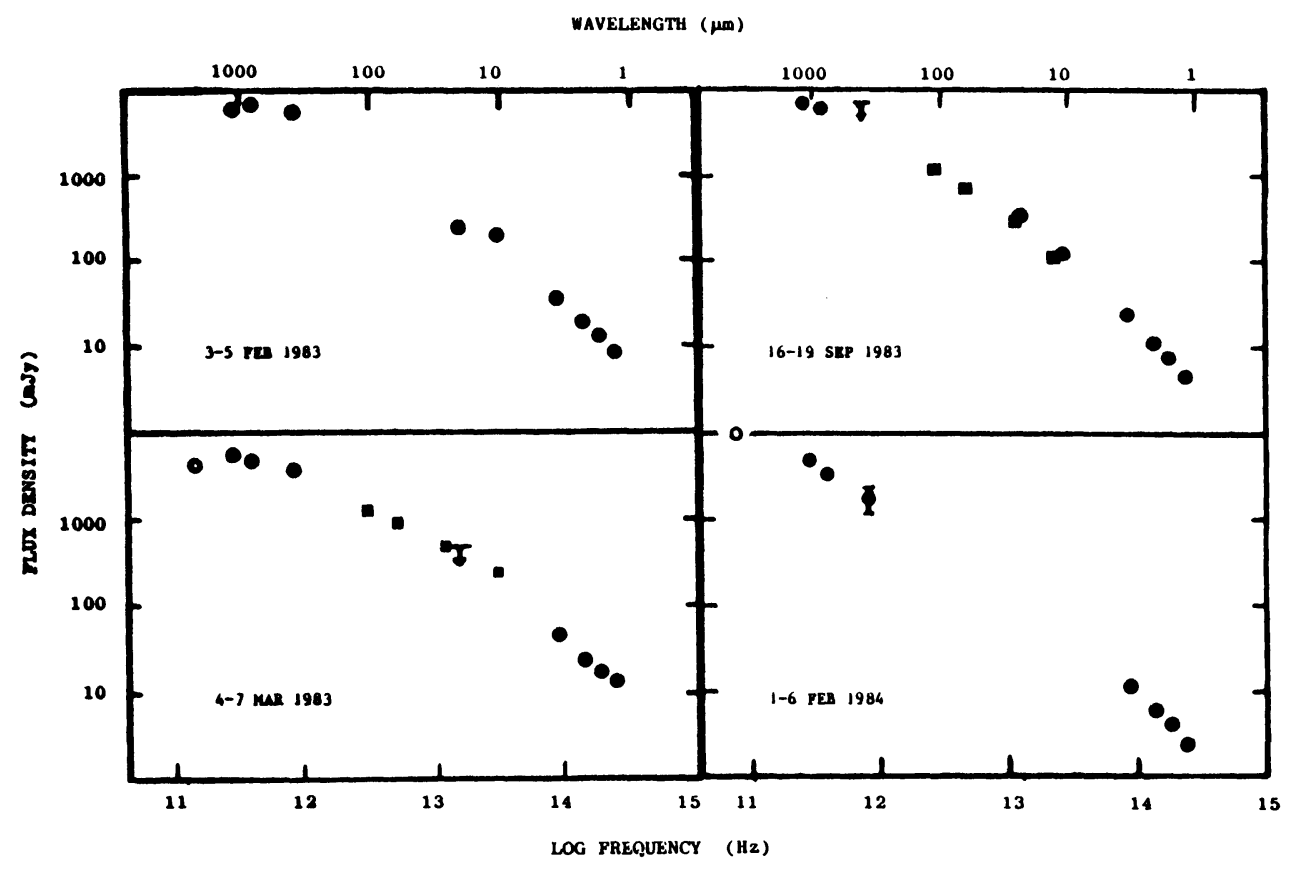

Fig 3. A one-year sequence of 'simultaneous' observations of the OVV quasar $1641+399$ (3C345). The IRAS points are shown by and apart from September are taken from Bregman et al (6). The turnover frequency can be seen to evolve to lower frequencies and increase in flux over the monitoring period. 
relativistic jet. The emergent spectrum evolves through three distinct phases corresponding to Compton, synchrotron and adiabatic expansion losses being dominant. The behaviour of the three sources conforms to the model predictions for the synchrotron stage. A specific prediction is therefore that new superluminal knots will emerge from the cores of 0J287, 3C279 and 3C345 in the next few years. The size of the shocked emitting region in the jet is calculated to be a thin disc of radius about $10^{-2}$ to $10^{-3} \mathrm{pc}$ and the magnetic field of order $0.1 \mathrm{G}$. An extensive 20 year span of observations of 3C345 has been pubished by Bregman et al (6) and we come to broadly the same conclusions, particularly with regard to the flattening and steepening of the IR spectrum during flaring and the various breaks which we both attribute to synchrotron radiative losses. In addition, the deductions of scale sizes and magnetic field for the emitting region are not wildly dissimilar in spite of different models being used to interperet the data.

An example of our wide wavelength monitoring is given in (11) for the OVV quasar 3C446 (2223-052). Our data were combined with those of Barbieri et al (12) to investigate the radio through X-ray behaviour of the source. It is seen that the entire infrared through UV region shows strongly correlated variability, the well-observed IR and optical fluxes varying together at least on a timescale of weeks or less. The entire IR-UV regime can be fitted by a relativistic jet model and the flare and post flare behaviour is explained by either reacceleration or new injections of electrons.

\section{The Enigmatic Quasar 3C273}

We originally attributed the overall radio through IR spectrum to synchrotron emission from a Marscher-type (13) relativistic jet. There was no evidence for IR through $\mathrm{mm}$ variability in 3C273 $(14,15)$ and we referred to the spectrum of Fig $4 \mathrm{a}$ as the 'quiescent' state. During 1983 we observed a major flare in the IR through mm emission (15) and subsequent monitoring showed the spectral development of this flare (Fig 4b). The IR flux increased by a factor of four, the submm flux trebled, $\nu_{m}$ increased and then evolved to lower frequencies at approximately constant flux density. The IR fluxes then decayed back to the 'quiescent' spectrum in a matter of months with the mm and submm fluxes decaying much more slowly. The flare luminosity exceeded $10^{12} \mathrm{~L}_{\circ}$ (assuming isotropic emission). The spectral development of the flare was well monitored and led to the production of the new model of Marscher and Gear (10). During 1985 the $1-4 \mu \mathrm{m}$ IR spectrum remained constant to within $5 \%$ of the 'quiescent' values. In January 1986, we found, to our surprise, that the millimetre and submillimetre fluxes (unobserved for a year) had fallen to a level almost a factor of three below the 'quiescent' state. Further observations between $10 \mu \mathrm{m}$ through $3.3 \mathrm{~mm}$ confirmed the decline well below the 'quiescent' level at all

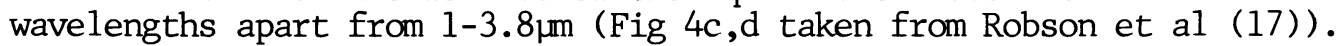
We are therefore forced to conclude that the optical through near IR spectrum has a different origin to that at longer wavelengths. The long 


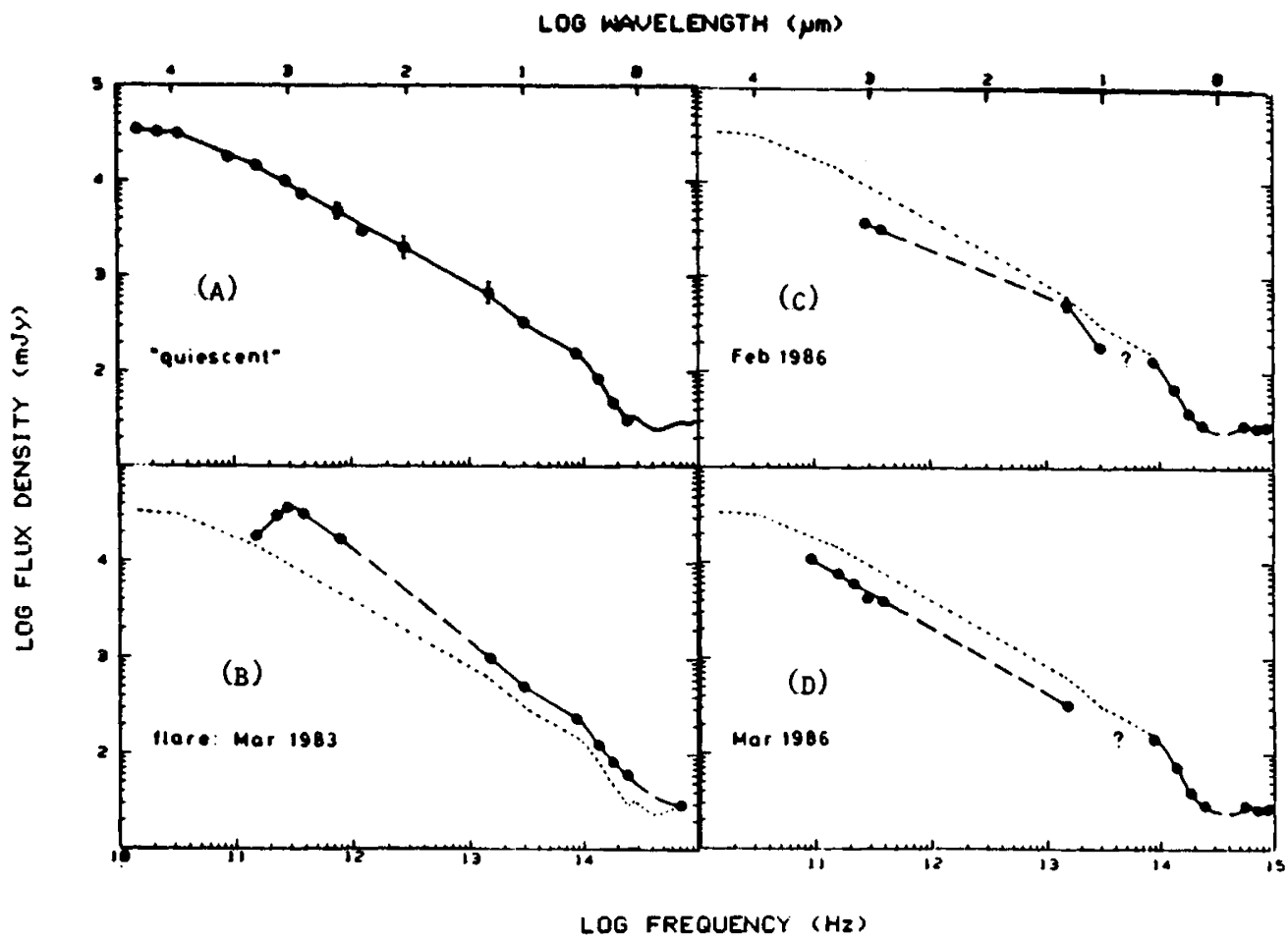

Fig 4. The spectrum of the quasar $1226+023$ (3C273) in four phases:

(A) the 'quiescent'; (B) the peak of the 1983 flare; (C \& D) the decline below the quiescent as observed in 1986, Ref 17. Note the non-variability of the IR component in C \& D.

wavelength spectrum is undoubtedly synchrotron in origin, but the source of the optical through IR spectrum is not clear (as is the case for all low polarization radio quiet quasars). It is very difficult to see how it can be of synchrotron origin and yet be so non-variable as a self-absorption turnover at about $5 \mu \mathrm{m}$ would give a source size of a few $\mathrm{AU}$ and a magnetic field of about $100 \mathrm{G}$ !. In addition, Courvoisier et al (18) show that the variability from radio through X-ray wavelengths in 3C273 strongly suggests the presence of at least four or five distinct components.

$3 \mathrm{C} 273$, the first quasar discovered, continues to give surprises. However our recent revelation that the optical/IR spectrum is distinct from the radio-loud synchrotron part, brings many of the differences between radio-loud and radio-quiet quasars into sharp perspective. All the results discussed above clearly show the value of coordinated multifrequency observations, particularly in the millimetre through $\mathrm{X}$-ray regime. The requirements for an X-ray observatory for programmes of variable extragalactic sources must be a high priority for the scientific comminity. 


\section{REFERENCES}

1 Robson,E.I. 1983 RAL-85-086, ed Gondhalekar

2 Gear,W.K. et al 1984, Ap.J., 280, 102.

3 Clegg,P.E. et al 1983, Ap.J., 273, 58

4 Gear,W.K. et al 1985, Ap.J., 291, 511

5 Gear,W.K. et al 1983 Nature, 303, 46.

6 Bregman,J.L. et al. 1986 Ap.J. 301, 708,

7 Gear,W.K. et al 1986 Ap.J., 304,295.

8 Brown,L.M.J. et al 1986 in preparation

9 Gear, Robson \& Brown 1986 submitted to Nature.

10 Marscher \& Gear 1986 Ap.J., 298,114

11 Brown,L.M.J. et al 1986, MNRAS, 219, 671

12 Barbieri,C. et al 1985 Ast.Astrophys. 142,316

13 Marscher,A.P., 1980, Ap.J., 235, 386.

14 Neugebauer,G. et al 1979, Ap.J. 230, 79.

15 Ennis, Neugebauer, \& Werner 1982, Ap.J., 262, 460.

16 Robson,E.I. et al 1983, Nature, 305, 194.

17 Robson,E.I. et al 1986, Nature, in press

18 Courvoisier,T.J.-L et al. in preparation

\section{DISCUSSION}

N.Bochkerev: Do I understand you to say that variations occur in the millimetre region on timescales of less than one month ? If this is correct what is the shortest timescale and the resulting amplitude of the variability?

Reply: We do see variations on this timescale and they have been up to nearly a factor of two. The best documented was the flare in 3C273 - see ref 16 above.

M.J.Ward: Is your latest near infrared variability data on 3C273 consistent with the hypothesis that the bump around $3.5 \mu \mathrm{m}$ is of thermal origin?

Reply: A thermal origin is still possible from the lack of variability of the near IR component. However it is not our most preferred solution to the puzzle of this part of the continum emission from $3 \mathrm{C} 273$ as the required temperatures exceed the evaporation temperatures for the dust. 